\title{
Cell per Dish
}

National Cancer Institute

\section{Source}

National Cancer Institute. Cell per Dish. NCI Thesaurus. Code C73637.

A unit of cell concentration expressed as a number of cells per a petri dish. 